\title{
Nuclear Membrane and Cytoplasmic Lamellae at Meiotic Prophase of Lilium Microsporocytes
}

\author{
S. K. Sen ${ }^{1}$ \\ Max-Planck-Institut für Züchtungsforschung, 5 Köln-Vogelsang, \\ Federal Republic of Germany
}

Received April 1, 1969

\section{Introduction}

All organisms except viruses, bacteria and blue-green algae, have the nucleus being separated from the cytoplasm by two concentric membranes. Except during certain phases of cell division, the nucleocytoplasmic interaction must proceed through this structure. It now seems certain that the nuclear membrane is formed from material already present in the cytoplasm during telophase. The nuclear membrane with its conspicuous pores and annuli is usually distinguishable from other membranous structures and the structure of the nuclear envelope has been observed to be remarkably uniform throughout the animal and plant systems. Certain cell types regularly possess cytoplasmic membranes (periodic lamellae or annulate lamellae) identical to the nuclear membrane but not in direct continuity with them. Their derivation from the nuclear envelope has also already been indicated. Cytoplasmic annulate lamellae in somatic cell are much less extensively developed than in germ cells.

In course of a series of observation on differentiation of meiotic prophase nucleus of Lilium in vivo and in vitro in presence and absence of DNA. inhibitor, certain observations on the ultrastructural details of nuclear membrane and their breakdown have been made, which are included in this text.

\section{Materials and methods}

Leptonema and zygonema microsporocytes of lily variety, Enchantment Orange were cultured (Sen 1969) with or without the presence of $2 \times 10^{-5} \mathrm{M}$ mitomycin C. Microsporocytes collected from plants grown in greenhouse did not reveal any ultrastructural differences from those that were grown in vitro without mitomycin $\mathrm{C}$. The rate of meiosis in the microsporocytes in vitro had been slightly slower than the rate at in vivo. However with mitomycin the rate of meiosis had reduced to almost half.

For electron microscopy materials were fixed in glutaraldehyde followed by $\mathrm{OsO}_{4}$ essentially as described by Ledbetter and Porter (1963). The tissues.

1 Permanent Address: Department of Genetics and Plant Breeding, Banaras Hindu University, Varanasi-5, India. 
were embedded in Veslopal $W$ and ultra-thin sections were made with Porter Blum microtome which were stained in uranyl acetate (Watson 1955) and lead citrate (Reynolds 1963). Materials were examined under Siemens Elmiskop I at $80 \mathrm{kV}$. Histochemical tests for the presence of RNA (Ruthmann 1958) were conducted with vestopal embedded tissue. Both toludine blue and azure B were used as stain. Successive sections were used for light and electron microscopy to keep a relatively close correlation between the distribution of basophilia and the fine structures of the same cell. Crystalline ribonuclease dissolved in distilled water has been used for enzymatic digestion.

\section{Results}

Structurally the nuclear membrane consists of two parallel membranes approximately $300-400 \AA$ apart. In tangential sections the pores are best revealed (Fig. 1). The diameter of pore in such sections in somewhat variable ranging from $600-700 \AA$. It is noted that the pore region is traversed by a membrane of greater density (Fig. 1). It is clear however from the density of material seen to be associated with the pores of nuclear envelope that some kind of material is associated with the pores (Figs. 1, 2, 3). In many cases, a homogenous matrix of considerable electron density appears to form a collar or ring around the inside of the pores and to extend a short distance from the pores. Although the high density associated with the pores makes it difficult to clearly reveal the complete structure of this region, it has been realised that the nuclear pores are octagonal (Figs. 1, 2,3) in shape, although in tangential sections the pores appear as ring-shaped structures. The dense circular annular material form a cylinder through the periphery of the pore and may extend for short distances above and below the nuclear envelope. That each pore has eight microcylinders (Figs. 1,2,3) along the annular wall and a small dense granule (Fig. 1) is observed in the center of the annulus have also been revealed. In untreated pachynema nucleus with intact nuclear membrane, some scattered pore complexes have been seen both inside and outside the nucleus (Figs. 2, 3). Pore complexes may be detached from the nuclear envelope during breakdown of the envelope. Some of these detached pores maintain to have outer annular rings with them (Fig. 3). The outer layer of the nuclear envelope at pachynema has been observed to actively engage in blebbing (Fig. 5) process. In some areas along the nuclear membrane and also in the cytoplasm series of small vesicles (Figs. 5, 6) have been observed. At late pachynema to 'diffused stage' (Moens 1968) the nuclear membrane starts to disintegrate, although at late pachynema beginning of ruptures to form have been observed in some regions of nuclear envelope. In untreated microsporocytes it has not been possible to follow the detailed process of breakdown of nuclear membrane as has been in the case of mitomycin $\mathrm{C}$ treated microsporocytes.

At diffused stage in mitomycin treated meiocytes, triple layered unit 


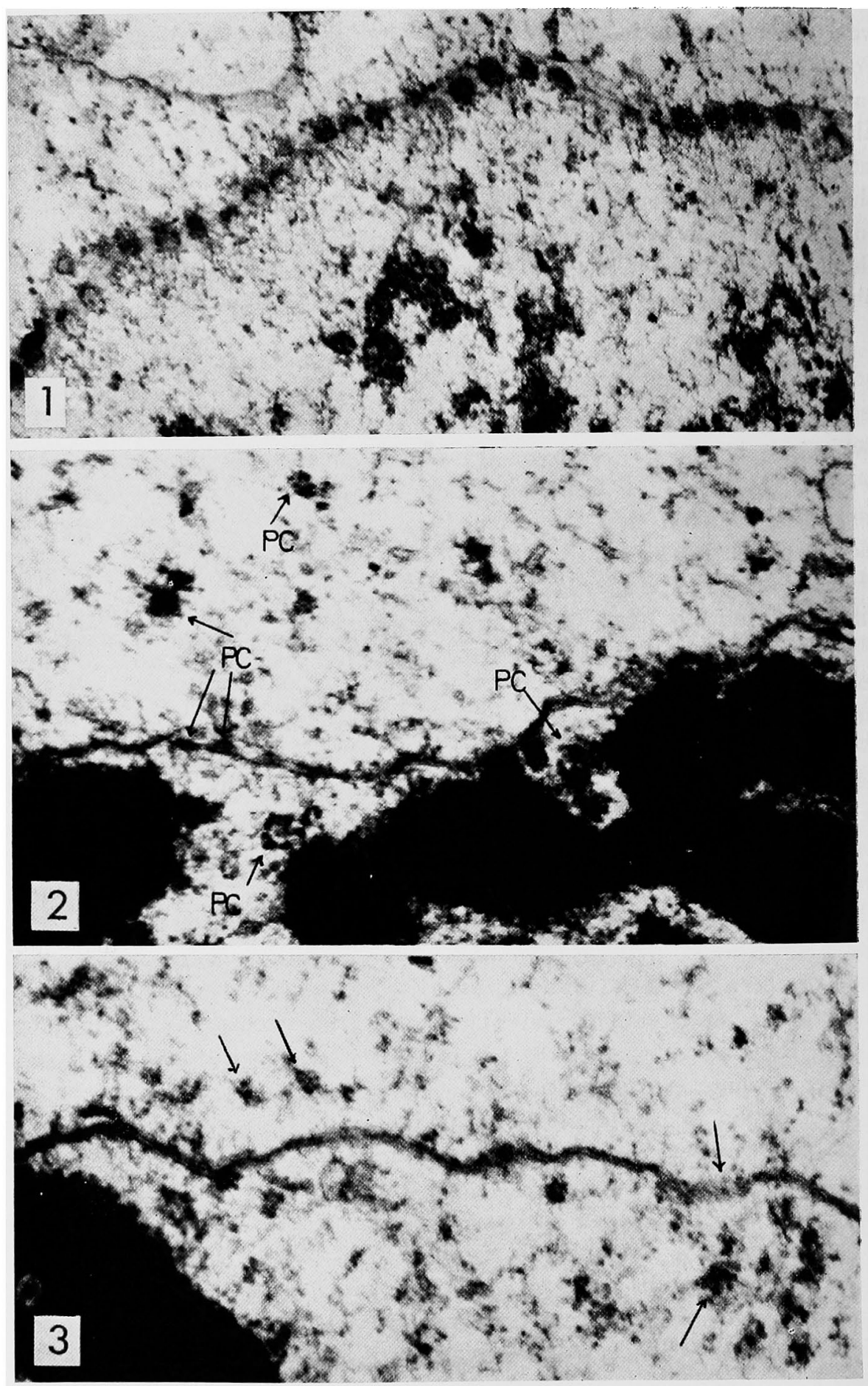

Figs. 1-3. 1, tangential section of nuclear membrane at early zygonema showing pore complex. $\times 42,000.2$, nuclear membrane at late pachynema showing nuclear pores (arrows) and detached pore complexes $(\mathrm{pc}) . \times 50,000.3$, nuclear membrane at late pachynema showing pore complexes (arrows). $\times 47,500$. 
membranes have been seen sometimes to dissociate from the chromatin (Figs. $4,7)$ situated near the nuclear envelope. These unit membranes have been consisted of two dark lines separated by a lighter line, each of these three regions is approximately $30-40 \AA$ thick (Figs. $4,7,8,9$ ). When are not outstretched they have a dimension of $200-300 \AA$. In small pieces (Fig. 8)
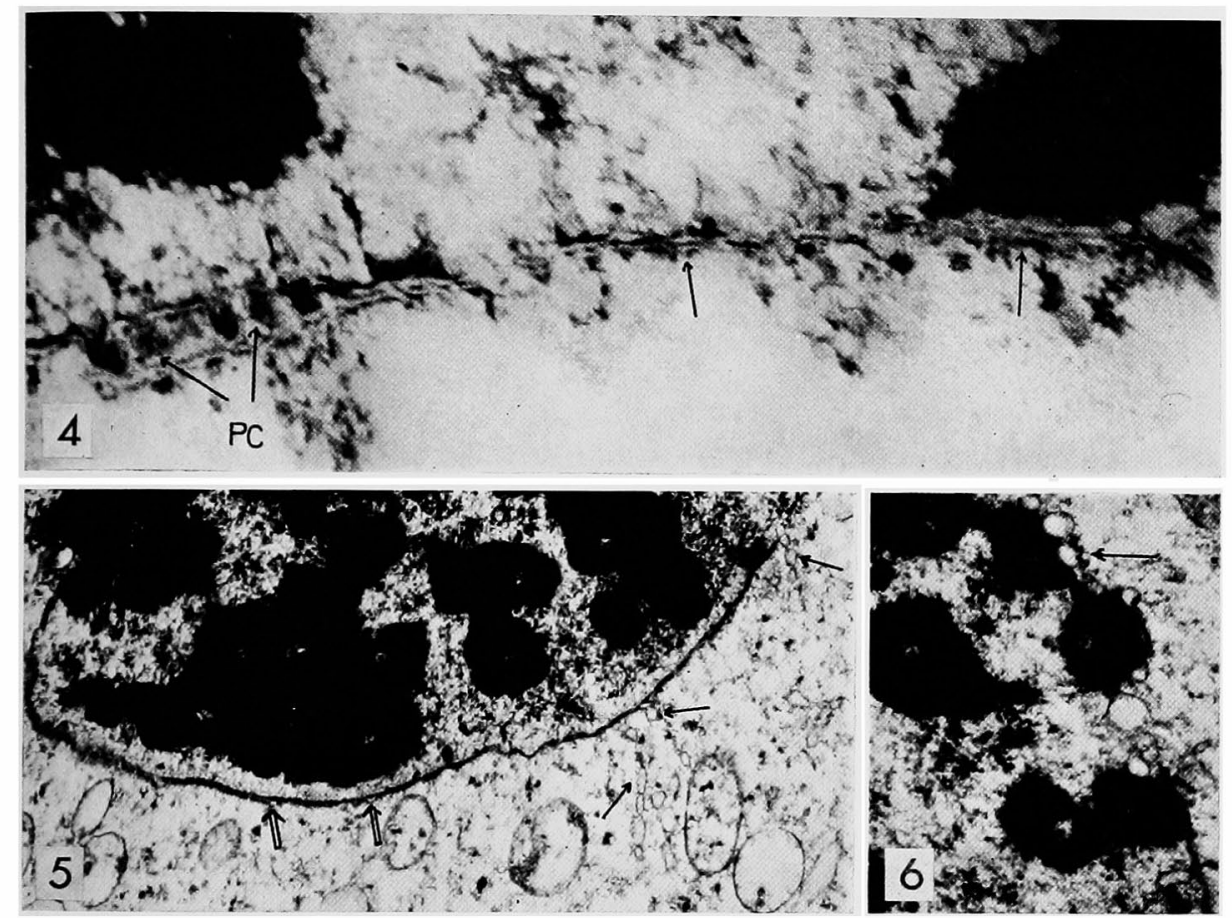

Figs. 4-6. 4, triple layered unit membrane at diffused stage in the process of being detached from chromatin when treated with mitomycin. Details of unit n.embranes can be seen (arrows) in presence of certain pore complexes (pc). $\times 33,300$. 5, late pachynema nuclear membrane showing vesicles (arrows) and blebbing (double-lined arrows). $\times 9,600$. 6 , late pachynema with rupture nuclear membrane with series of vesicle.

they have been seen later to be collected into groups of 3-4 per cell of cytoplasmic lamellae (Figs. 9, 11). These membranes which are collected into groups of lamellae apparently appeared as comparable to annulate lamellae, although the annular regions are not as clear as have been previously accounted in other germ line systems. Two structural components have been seen between the lamellae (Figs. 9, 10, 11). These components are a small dense granule and an exceedingly thin filament. Mostly the granules are attached to the thin filaments. The lamellae do stain basophilic. It is suggested further that these granules $(30-40 \AA)$ may represent a kind of RNA or RNP probably not similar to the cytoplasmic ribosomes. The granular material often shows a specific orientation (Fig. 9). There has also been varied concentration of granules in some regions of the lamellae (Fig. 11). The interlamellar regions. Filled with filaments and granules have not shown any preferential 


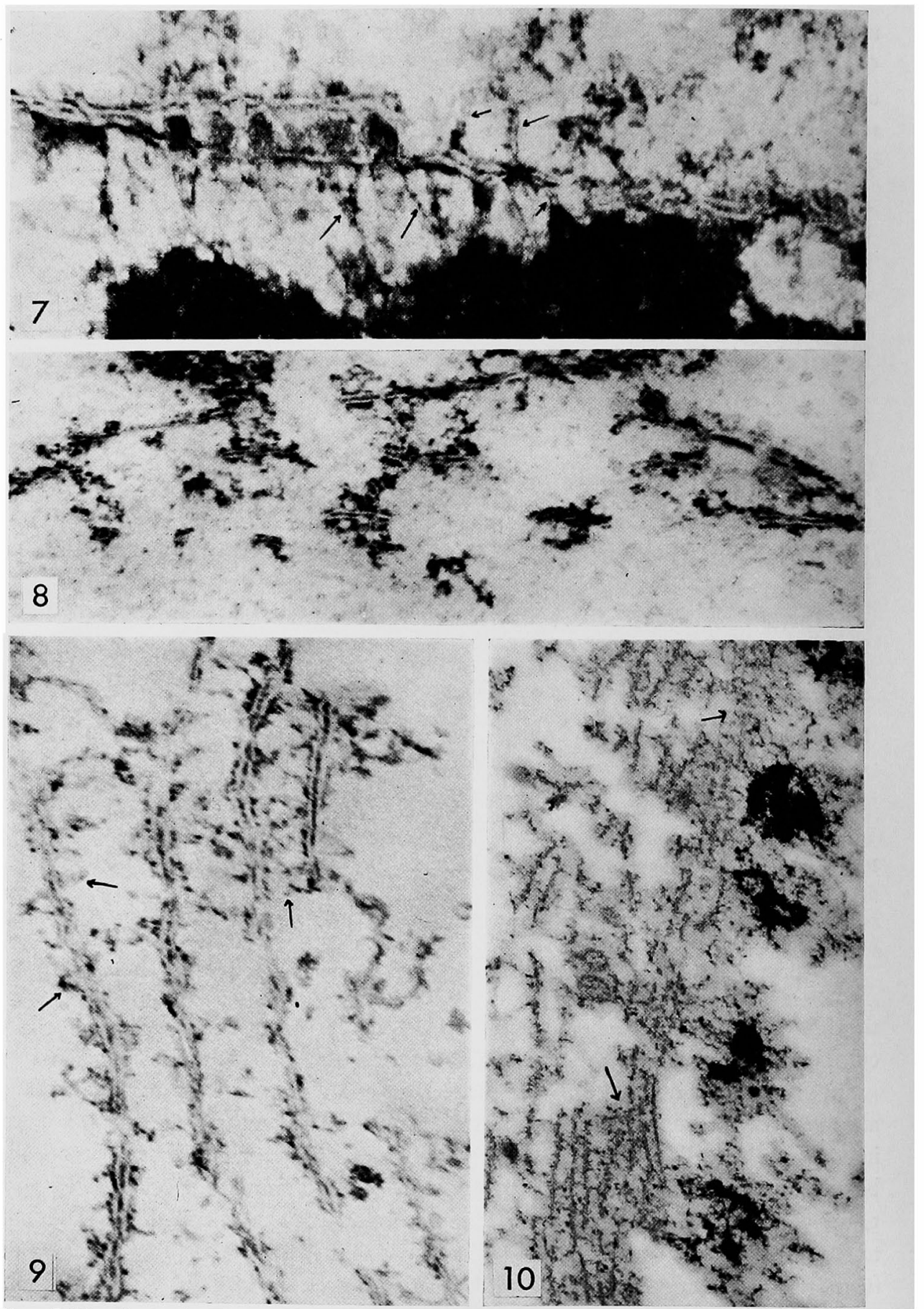

Figs. 7-10. 7, continuation of Fig. 4. It shows fine fibrous connections of unit membranes with chromatin. $\times 51,200$. 8 , at diffused stage mitomycin treated meiocytes revealed fragments of unit membranes. $\times 33,3009$, cytoplasmic lamellae with granules and filaments at diffused stage of mitomycin treated meiocytes show specific orientation (arrow). $\times 43,500$. 10, chromatin and lamellae surrounded with granules and filaments at diffused stage of mitomycin treated meiocytes. Arrows indicate that the lamellae are triple layered. $\times 9,600$. 
alignments with respect to any structure on the lamellar system. Sometimes at diffused stage, chromosomes have revealed to be surrounded with granules and filaments (Fig. 10), similar in nature as they have been found in associ-
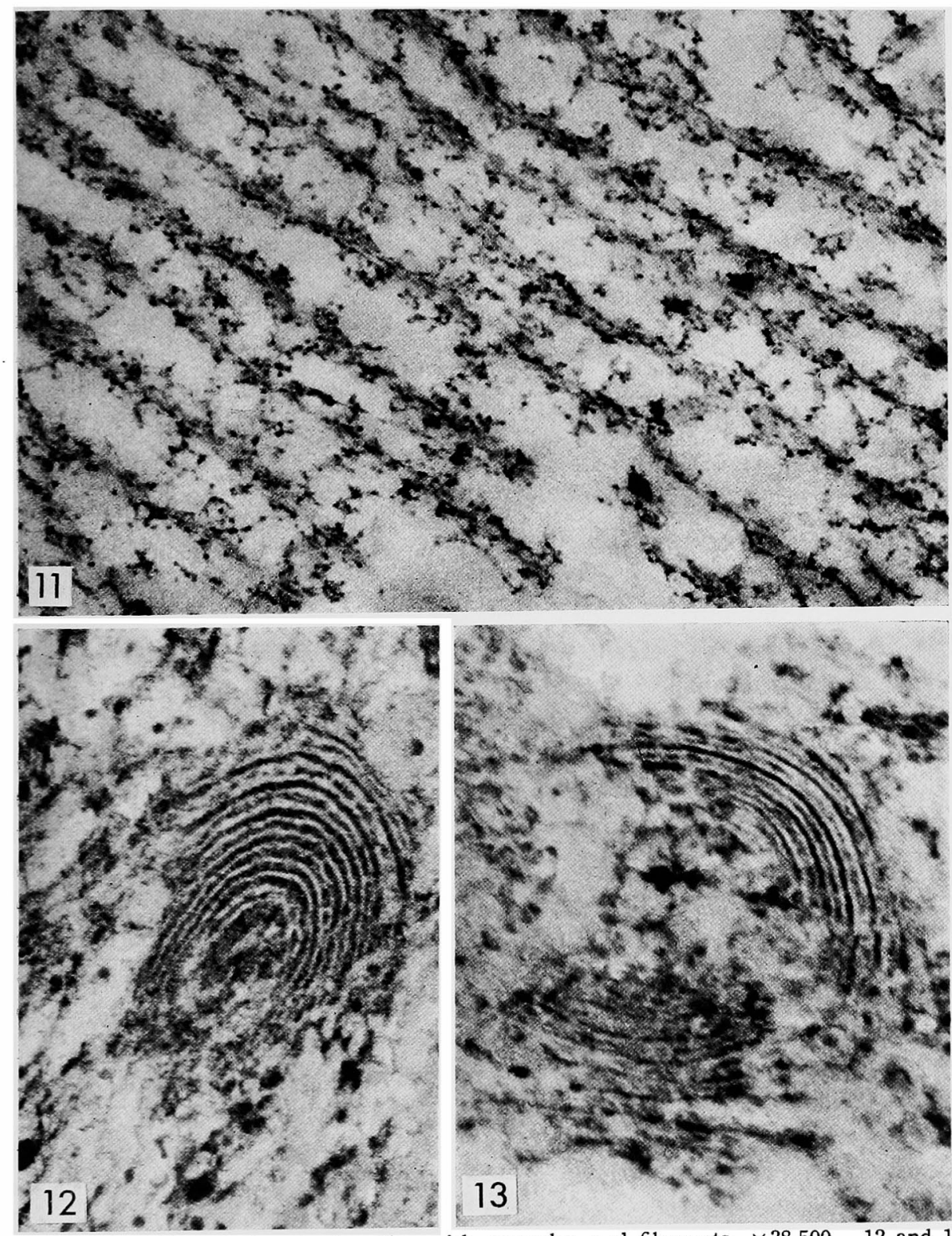

Figs. 11-13. 11, cytoplasmic lamellae with granules and filaments. $\times 38,500.12$ and 13 , tubular ellipsoid lamellae. $\times 77,000$ and 64,000 resp.

ation with neighbouring lamellae. These cytoplasmic lamellae later arrange into stacks and change over to tubular ellipsoid forms (Ruthmann 1958) varying in sizes. They have been always found in the plasma away from 
the chromatin. At diplotene no traces of such lamellae have been seen in these cell systems. No special affinity for endoplasmic reticulum (ER) has been observed to these lamellae. The ellipsoid stacks are distinguishable from the typical golgi complex seen in the plasma, so are the lamellae from the ER.

The association of the triple layered unit membrane at diffused stage when treated with mitomycin have revealed that portions of chromatin are being stretched (Figs. 4,7) by fibrous connections. These fibrous connections are of similar in dimension of the filaments seen with the granules with the lamellae. The significance of this is difficult to estimate, although it perhaps indicates towards the association of chromatin with the nuclear membrane during the earlier stages of the meiotic prophase which is lost due to the breakdown of nuclear membrane. In addition to this, some pore complexes (Figs. 4,7 ) have been seen to be loosely placed in between two such lamellae. A definite relationship between these three-lined elements to nuclear membranes has not been possible to establish.

\section{Discussion}

The idea of octagonal pore complex first suggested by Wischnitzer (1958) and later demonstrated by Gall (1967) has been found to be acceptable. The occurrence of annulus associated with the dissociated pore complex indicate that the association of pores to the membranes may be through annulus region which is round in shape. Hence, the nuclear pore may be round in shape (Wischnitzer 1958) rather than octagonal (Gall 1967).

The source of origin of the three-lined unit membranes which have been found to aggregate to form cytoplasmic lamellae has not been possible to establish. The unit membrane of Robertson (1959) has been widely demonstrated in variety of membranes in living systems. At times each of the membranes of the nuclear envelope may show evidence of triple layered condition (Gall 1964) which has been thought to be probable in this case. The dimension of these unit membranes is probably little too large to fit into a functional nuclear membrane in the same system. Again this may not be the case as these membranes may reveal their ultrastructures only when they are loose and also when they are not transformed into the rigid nuclear envelope.

The best evidences indicate that the membranes used in the construction of annulate lamellae is derived from the outer layer of the nuclear envelope in the case of cytoplasmic annulate lamellae (Kessel 1968). Till now evidences support that structurally nuclear envelope and annulate lamellae are similar. Although a clear mechanism of the origin of lamellae from the nuclear envelope has not been possible to demonstrate. Swift's (1956) suggestions on the possible mechanism for the origin of annulate lamellae by fragmentation of the nuclear membrane and or by synthesis of annulate 
lamellae on the nuclear membrane with the nuclear membrane acting as a template, are acceptable. Likewise Rebhun's (1961) postulation on delamination process of entire nuclear envelope is also indicative, particularly when the single lamellar systems have been seen to breakdown to form cytoplasmic lamellae. Series of vesicles have been seen to form at late pachynema, although any relationship between blebbing of outer membrane of nuclear envelope and vesicles has not been observed. The most widely adopted hypotheses on the origin of the annulate lamellae are that they are derived from the membrane sloughed off from the nuclear envelope (Kessel 1963, 1964a, b, 1965). Role of vesicles as outlined and documented by Kessel (1963, $1964 \mathrm{a}, \mathrm{b}, 1965,1968)$ and later also suggested by Harrison (1966) is worth considering.

The fact of presence of cytoplasmic lamellae only at the time of degeneration of nuclear membrane indicates that their occurrence is somehow related to the elements of nuclear membrane. The membraneous systems observed in this system is morphologically differentiable from the ER. While the three layered character of the unit membrane, presence of small granules and filaments, their restricted distribution and number and their different forms make them different structurally from ER. The tubular ER in spermatogonia of grasshopper (Sakai and Shigenaga 1967) cannot be compared with the tubular lamellae in the present system as the latter's origin points to an another membranous system. This membranous system can as well be very close to ER system, as the relationship of annulate lamellae and nuclear envelope to ER is almost an established fact. Moreover the cytoplasmic lamellae observed here in this experimental system cannot probably be strictly called annulate lamellae as they are not as well developed as they have been often seen (Kessel 1968) in other systems. The cytoplasmic lamellae when are arranged into stacks, they change over to tubular forms, which are comparable to tubular lamellae observed in crayfish spermatocytes (Ruthmann 1958).

Presence of RNP particles associated with cytoplasmic lamellae and their timely disappearance may suggest of a biochemical machinery necessary for the functional activity of this cytoplasmic membrane system. The migration of the lamellae in the cytoplasm carries out nuclear derived information to the remote regions of cytoplasm has been suggested earlier (Moses 1964, Swift 1968). One or more large stacks of annulate lamellae in a cell could provide the framework for the aggregation of specific raw materials for the construction of a special group of compounds at certain states of differentiation (Kessel 1968). The basophilic nature of the annulate lamellae and close association of RNA may reflect in the genetic transfer of genetic specificities from the nucleus to the cytoplasm (Ruthmann 1958, Swift 1956). The significance of the granules and filaments around the chromosomes is difficult to speculate. 
Experimental studies carried out by earlier workers have revealed that the formation of annulate lamellae is perhaps an expression of cell proliferation (Hruban, Swift and Slesers 1965). Indications through abnormal developmental processes (Stay 1965, Merkow and Leighton 1966, Baker and Franchi 1967, Doolin 1967), reduced temperature development (Merkow and Leighton 1966) and hormone treatments (Dwichow, Boily and Dhainant 1965, Dhainant 1966) may as well suggest that the occurrence of annulate lamellae might be a morphological indication of abnormal protein synthesis and perhaps also suggest that the cerebral hormone may be regulator of DNA and RNA synthesis.

In the present system it is known (Ito, Hotta and Stern 1967) that the level of mitomycin tried, prevents a substantial amount of DNA synthesis at zygonema and pachynema and the rate of meiosis is considerably reduced. Considering our present knowledge the way protein is synthesised, the mode of action of mitomycin is difficult to speculate. The breakdown of nuclear envelope and their differentiation into lamellar system be as well quite a normal short-life process which has not been possible to observe in the nontreated meiocytes. On the otherhand this can again be an abnormal phenomenon for this cell system which may be normal to others as in many normal systems annulate lamellae have been radily observed. In plant system such lamellae have not been earlier reported.

\section{Summary}

The ultrastructure of nuclear membrane and cytoplasmic lamellae have been studied at meiotic prophase in the microsporocytes of Lilium in vivo and in vitro in presence and absence of mitomycin C. Meiocytes were fixed in glutaraldehyde and $\mathrm{OsO}_{4}$ and sections were stained in uranyl acetate and lead citrate. The nuclear pores have been found to be octagonal in shape with eight microcylinders. The pore complexes are probably detached from the nuclear wall during the breakdown of the latter. At diffused stage in the mitomycin treated meiocytes triple layered unit membranes have been seen to form cytoplasmic lamellae. Two structural components, small dense granules and exceedingly thin filaments, have been seen always to be associated with these lamellae. These lamellae stain basophilic. They later arrange into stacks and change over to tubular ellipsoid forms. Sometimes these threelayered unit membranes have been seen to dissociate from chromatin at the time of the breakdown of the nuclear wall. The significance of the formation of these cytoplasmic lamellae has been discussed.

The author thanks Prof. J. Straub, Dr. I. Anton-Lamprecht for their interest in this study and to Mrs. S. Sen, Miss B. Meyer and Mr. D. Bock for help. Financial grants from Alexander von Humboldt Stiftung and MaxPlanck-Gesellschaft are acknowledged gratefully. 


\section{Bibliography}

Baker, T. G. and Franchi, L. L. 1967. J. Cell Sci. 2: 213.

Dhainaut, A. 1966. Compt. Rend. Soc. Biol. 160: 749.

Doolin, P. F. 1967. Anat. Record. 157: 236.

Durchon, M., Boily, B. and Dhainaut. A. 1965. Compt. Rend. Soc. Biol. 159: 106.

Gall, J. G. 1964. Protoplasmatologia. 5: 4.

- 1967. J. Cell Biol. 32: 391.

Harrison, G. A. 1966 . J. Ultrastruct. Res. 14: 158.

Hruban, Z., Swift, H. and Slesers, A. 1965. Cancer Res. 25: 708.

Ito, M., Hotta, Y. and Stern, H. 1967. Develop. Biol. 16: 54.

Kessel, R. G. 1963. J. Cell Biol. 19: 391.

- 1964a. J. Ultrastruct. Res. 10: 498.

- 1964b. Z. Zellforsch. 63, 37.

- 1965. J. Cell Biol. 24: 471.

- 1968. J. Ultrastruct. Res. 10 (Suppl): 1.

Ledbetter, M. C. and Porter, K. R. 1963. J. Cell Biol. 19: 239.

Merkow, L. and Leighton, J. 1966. J. Cell Biol. 28: 127.

Moens, P. B. 1968. Chromosoma 23: 418.

Moses, M. J. 1964. In Cytology and Cell Physiology (G. Bourne, ed) 424, Acad. Press, New York.

Rebhun, L. I. 1961. J. Ultrastruct. Res. 5: 208.

Reynolds, E. S. 1963. J. Cell Biol. 17: 208.

Robertson, J. D. 1959. Biochem. Soc. Symp. 16: 3.

Ruthmann, A. 1958. J. Biophys. Biochem. Cytol. 4: 267.

Sakai, A. and Shigenaga, M. 1967. Cytologia 32: 72.

Sen, S. K. 1969. Nature 224: 178.

Stay, B. 1965 . J. Cell Biol. 26: 49.

Swift, H. 1956. J. Biophys. Biochem. Cytol. 2, 415.

- 1968. Symposium on the chemical basis of development, John Hopkins Press, 174 (Baltimore, Maryland).

Watson, M. L. 1955 . J. Biophys. Biochem. Cytol. 1: 257.

Wischnitzer, S. 1958. J. Ultrastruct. Res. 1: 201. 